\title{
Evaluating Translation Quality via Utilizing Skopos Theory
}

\author{
Cai Ning \\ College of Electrical Engineering \\ Northwest University for Nationalities \\ Lanzhou, China \\ E-mail: caining91@tsinghua.org.cn
}

\author{
Zhou Jian* \\ College of Electrical Engineering \\ Northwest University for Nationalities \\ Lanzhou, China \\ E-mail: 397125172@qq.com \\ *Corresponding Author
}

\begin{abstract}
It is known that, the most important element in the translation activity is the target audience. Based on the 'Skopos theory', this study will analyze the quality of translation of Harry Potter and the Philosopher's Stone from People's Literature Publishing House in 2000. From the advantages and disadvantages of previous works, it is safe to find the basic skills for translation. Also, the survey of readers' experience will make the result more convincing. It is a qualitative and descriptive study by analyzing those translation examples. However, in the main body part, some simple methods, like detailed explanations and analysis will be used to support the author's point of view with more details, with the hope to make the findings more convincing. As for the content of study, four-character words make the whole story full of rhythm, the translation of Chinese idioms helps Chinese readers understand the meaning deeper, and the translation of sentences is important and common.
\end{abstract}

Keywords-Translation; Target Audience; Skopos Theory; Quality; Idiom

\section{INTRODUCTION}

It is known that, the most important element in the translation activity is the target audience. Some of the researchers oriented the target as children, so they defined the Harry Potter and Philosopher's Stone as children's literature. So they conclude the translation activities as: First, children for Harry Potter prefer concrete words rather than formal and abstract ones, so when the translators are translating the stories, they use more concrete words to make children have a deep understanding. Second, vivid expressions are better than plain ones for children to read. Translators can use rhetorical devices such as metaphors, pun, and simile etc. to enrich the beauty of the text. Third, translators should try their best to maintain the musicality and readability of the original text to satisfy readers' unique aesthetic tastes. Fourth, to translate for children, translators should adopt domestication as a primary strategy which can help children be familiar with what they read and get involved in the plot. Last but not least, translators should use adaptation in order to accommodate target child readers' capability of understanding. In the process of translation, the translator should first take the children as a basis reader, standing in the position of children, maintain the original feeling from young readers [1]

The Chinese translations have two versions: The mainland version and the Taiwan traditional versions. The mainland readers and Taiwan readers share the same language but have different understanding of the story clue. The Researchers have studied these two versions separately, and compared them.

Compared with the translation of Taiwan version, the mainland version published by People's Literature Publishing House is more commercialized and more close to the readers. The translators translate Harry Potter both for the young readers and for the adults, not only regarded as children's literature. The publisher has cast eyes on the market of all ages, regarding Harry Potter as the bestseller to all tastes [2].

Another researcher studied the difference between the culture in mainland and Taiwan, and tries to understand the two versions from the perspective of cross-strait culture. In this study, it is found the influence of culture and history to the translation of Harry Potter and Philosopher's Stone. It is shown that the mainland and Taiwan has different culture as well as culture background, so the translations are different not only in the simplifier and traditional characteristic, but also the way people understand it. So it can be concluded that the translators did the translations by considering the feeling of target readers. It corresponds to the key idea of Skopos Theory: the target audience has the highest priority [3].

Based on the 'Skopos theory', this study will analyze the quality of translation of Harry Potter and the Philosopher's Stone from People's Literature Publishing House in 2000. From the advantages and disadvantages of previous works, it is safe to find the basic skills for translation. Also, the survey of readers' experience will make the result more convincing. It is a qualitative and descriptive study by analyzing those translation examples. However, in the main body part, some simple methods, like detailed explanations and analysis will be used to support the author's point of view with more details, with the hope to make the findings more convincing.

The reasons why Harry Potter and the Philosopher's Stone is chosen to be the subject of this research can be listed as follows: Firstly, this book is considered to be the most influential book world-wide, which means it is worth being studied. Secondly, there are loads of previous researches that have been done in this field. This makes the whole study become expert-guided. Thirdly, due to its popularity, the original book and translations are relatively easy to be obtained in bookshops, which, to a large extent, will facilitate the researching process. Taking all the above 
factors into account, Harry Potter and the Philosopher's Stone is finally chosen as the subject for this study.

Harry Potter and the Philosopher's Stone from People's Publishing House in 2000 is the most popular version in the mainland which has a great influence in children literature in China.

As for the content of study, four-character words make the whole story full of rhythm, the translation of Chinese idioms helps Chinese readers understand the meaning deeper, and the translation of sentences is important and common.

\section{AN OVERVIEW OF SKOPOS THEORY AND ITS APPLICATION IN TRANSLATION}

The 'Skopos theory' is originated in Germany, and then it becomes widely accepted and recognized in modern translation study field. The meaning of Skopos in Latin means aim, purpose, intention or function. The key idea in Skopos theory is that the translation action is the way to deliver information, which means, the translations don't need to be word by word but need to present the key meaning from source text.

It is found that, translators always cannot deliver all the meaning by translating the source text word by word. When the translators highlight some of the meanings, the other parts of the source text always are suppressed. Then, it is not surprising that the translation activities always sacrifice parts of the original text [4]. Thus, for translators, it is important to decide which parts to keep and which parts to omit. The Skopos theory points out the audience is the most important role, so the purpose of the source text has the highest priority. The audience is the readers of translated text. The characteristic of the audience is they have their own background knowledge, the expectation of the target text and the requirement of the meanings [5]. In Harry Potter and Philosopher's Stone, the audience is the readers in Chinese mainland, most of the readers are children.

The 'Skopos theory' mainly has three principles, namely: Skopos Rule, Coherence Rule and Fidelity Principle, the Principle of Loyalty.

The first rule requires the translators to translate from the perspective of target language which puts the reader's reading experience in the first place. This rule has the highest priority in the whole theory. The process to achieve the purpose of translation determines the translation action itself, which means the results guide the method. There always are three purposes during the translation activities. The first one is the basic translation; the second one is the communicative purpose of the translation, such as inspire the readers; the last one is to use some special method to translation, such as to explain the special grammatical structure in a language. Mostly, the purpose is the communicative function. So the translator according to this to decide the translation methods: literal translation or free translation [6].

The second rule and the third rule is not generally suitable for every case, therefore, it is less important than the first rule. Coherence Rule means the target text must meet the intra textual coherence standard. In other words, the target text needs to require readability and acceptability [7].
Loyalty principle focuses on the relationship between the main concern in the process of translation, the translator and the author; the translator should follow the principle of "function plus loyalty" to improve the translation activities [5].

Another aspect of Skopos theory is regarded as "Adequacy but not Equivalence". To be more specific, the target text needs to satisfy the target audience but it doesn't mean the translator needs to translate word by word. The target text and the source text have the difference sentence but express the same meaning. Equivalence is not a "must" criterion to judge the translation. It is only the static form of dynamic Adequacy. So it is important to consider the potential function of the original text [8].

This section explained what the Skopos theory is and listed out important rules inside. The significant concept inside the theory is the priority of the target audience. In other words, the translators do not necessarily translate the source text word by word, but the more important part is the target text. Also the criteria of commenting a translation is not how it equals to the source text but how adequate and accurate it delivers the original meaning.

To conclude, 'Skopos theory' is an important theory in translation, and Harry Potter and the Philosopher's Stone is the most typical literature. So it is reasonable to take these two into research.

\section{REASONS FOR THE DIFFERENCES OF ANIMAL WORDS IN CHINESE AND WESTERN CULTURES}

\section{A. Overview}

The previous sections explained what the Skopos theory is and listed out important rules inside. The significant concept inside the theory is the priority of the target audience. In other words, the translators do not necessarily translate the source text word by word, but the more important part is the target text. Also the criteria of commenting a translation is not how it equals to the source text but how adequate and accurate it delivers the original meaning.

Besides, it is found that researchers study the translation from the children's literature perspective. Children's literature has a great influence on children's growth. It has listed eight functions of children's literature: to cultivate humanitarianism, that is, to have critical eyes, to hold rich imagination, to improve aesthetic sensibility, to abandon unease and to be intelligent and humorous, to develop inimitable idea, and to develop language skills [9]. After reading fairy tales children could broaden their vision. Books can help children grow in aspects of social, psychological, and cognitive development, including moral development and social learning. From all the statements above, it is found that previous studies lay more emphasis on the Children's literature and Harry Potter and the Philosopher's Stone. But in fact, this book is more than Children's literature; the author intends to deliver the idea to the adult. So in this paper, it will show more analysis from the aspect of ordinary people [10].

Researchers also did study on the comparison between the Taiwan version and Mainland version from the aspect of culture and history. In this thesis, it will present how the difference between Taiwan traditional version and 
mainland simplified version to comment on the translation version from People's Publishing House, China.

\section{B. Translation with Four-Character Words}

Words of four characters are quite common in Chinese, and most of them have their own original stories [11-15]. In the translation of Harry Potter and Philosopher's Stone, those four-character words are widely used. According to the literature review stated above the Skopos theory is emphasized on the Skopos Rule, which means it is required the translators translate from the perspective of target language which puts the reader's reading experience in the first place. The Four character words is the special in Chinese culture, which means by using these words as target text, the translator considerate the understanding of Chinese readers. These words help to express the meaning of the source text; what's more, they make the version quite close to Chinese culture. These make the translation correspond to the Skopos theory.

\section{Example:}

Enter, stranger, but take heed

Of what awaits the sin of greed,

For those who take, but do not earn,

(J. K. Rowling, 1997)

Qing-Jin, Mo-Sheng-Ren, Bu-Guo-Ni-Yao-Dang-Xin Tan-De-Wu-Yan-Hui-Shi-Shen-Mo-Xia-Chang, Yi-Wei-Suo-Qu, Bu-Lao-Er-Huo,$$
\text { ... }
$$

(N. Su, 2009)

The sin of greed means the guilt of being greedy, in the original story; this phrase is in a poem, which is translated as a four-character words "Tan-De-Wu-Yan". The form of this poem delivers the meanings with rhythm; the translation makes the style quite tidy and sounds good. So it corresponds to the Skopos Rule and Coherence Rule in the Skopo Theory.

Take, but do not earn was translated as two separate four-character-words. "Yi-Wei-Suo-Qu, Bu-Lao-Er-Huo", these words are concise and in the rhythm, which clearly describe the meaning as well as the negative emotion behind the sentence.

\section{Example:}

"No," he said finally. "No, we'll ignore it.

If they don't get an answer ... yes that's best...we won't do anything."

(J. K. Rowling, 1997)

"Bu," Ta-Zhong-Yu-Shuo, "Bu, Wo-MenGei-Ta-Lai-Ge-Zhi-Zhi-Bu-Li. Ru-Guo-TaMen-Shou-Bu-Dao-Hui-Xin...Dui, Zhe-Shi-ZuiHao-De-Ban-Fa...Wo-Men-An-Bing-Bu-

Dong...

(N. Su, 2009)

"We'll ignore it" is a simple sentence in the story, but the author has shortened it as a four-character word "ZhiZhi-Bu-Li". It makes the sentence tidy and short.

"An-Bing-Bu-Dong" means not change and wait until something happened. This phrase is always used in battles. Here in the original story, the author is trying to convey the battle feeling between speakers, so this phrase is correspondent to the context and expresses the traditional
Chinese culture. This Four-character Word makes readers feel familiar.

\section{Example:}

"......they're deep mind centaurs..... they know things......just don't let on much."

(J.K. Rowing, 1997)

"Zhe-Xie-Ma-Ren-Shen-Ao-Mo-Ce...TaMen-Zhi-Dao-Xu-Duo-Shi-Qing...Que-ZongShi-Shou-Kou-Ru-Ping."

(N. Su, 2009)

"Shou-Kou-Ru-Ping" means keeping the mouth close tight. This always means wise men say nothing to others. From the context, deep mind centaurs are those wise men that always keep their mouth and say nothing. This translation is short but precise, which makes sense to readers.

From these examples, we can see that for the proper use of these four-character words, the translators vividly use short sentences to express the exact meaning of the Source text, and make the readers of Chinese children feel like reading a typical Chinese Book.

\section{Translation of Chinese Idioms}

The use of Chinese idioms makes the version quite humorous and vivid. The following examples show the use of Chinese idioms:

\section{Example:}

Harry and Ron managed to get on the wrong side of him on their very first morning.

$$
\text { (J.K. Rowling, 1997) }
$$

Kai-Xue-De-Di-Yi-Tian-Zao-Shang, LuoEn-He-Ha-Li-Jiu-Gen-Fei-Er-Qi-Zhi-Jian-ChanSheng-Le-Jie-Di.

$$
\text { (N. Su, 2009) }
$$

In this sentence, 'get on the wrong side of somebody' means to make somebody unhappy. While from context, it can be found that in the target text, "Jie-Di" is used to describe unhappiness caused from a quarrel.

\section{Example:}

Strict and clever, she gave them a 'talkingto' the moment they sat down in her first class.

(J.K. Rowling, 1997)

"Ta-Yan-Ge, Cong-Ming, Ta-Men-GangZuo-Xia-Lai-Shang-Di-Yi-Tang-Ke-Ta-Jiu-GeiTa-Men-Lai-Le-Ge-Xia-Ma-Wei."

$$
\text { (N. Su, 2009) }
$$

In this example, 'talking-to' means blaming somebody. The Chinese idiom "Xia-Ma-Wei" makes the version more humorous and is easily accepted by Chinese children.

\section{Example:}

Harry was very relieved to find out that he wasn't miles behind everyone else.

$$
\text { (J. K. Rowling, 1997) }
$$

"Ha-Li-Fa-Xian-Zi-Ji-He-Da-Jia-Ye-BuGuo-Wu-Shi-Bu-Yu-Bai-Bu-Zhi-Cha, Yu-ShiDa-Da-De-Song-Le-Yi-Kou-Qi."

$$
\text { (N. Su, 2009) }
$$

The translator quoted "Wu-Shi-Bu-Yu-Bai-Bu" which exactly described that Harry did not fall behind other 
students. All of these typical Chinese idioms help Chinese readers get familiar with the target text.

\section{DISADVANTAGE OF TRANSLATIONS}

Despite the excellent translation cited above, there are also some disadvantages. The imperfect translations are sorted into different reasons. Some are due to the culture and historical difference.

\section{Example:}

"Mr Dursley always sat with his back to the window in his office on the ninth floor."

(J. K. Rowling, 1997)

"De-Si-Li-Xian-Sheng-Zai-Ta-Jiu-Lou-DeBan-Gong-Shi-Li, Zong-Shi-Xi-Guan-Kao-ZheChuang-Er-Zuo."

$$
\text { (N. Su, 2009) }
$$

It should be translated as "De-Li-Si-Xian-Sheng-ZaiTa-Shi-Lou-De-Ban-Gong-Shi-Li, Zong-Shi-Xi-GuanKao-Chuang-Er-Zuo", because the ninth floor in Britain is actually tenth floor in China due to the existence of "ground floor".

Another reason for the misunderstanding of translation is the multiple meaning of the words.

\section{Example:}

"Harry was used to spiders, because the cupboards under the stairs were full of them."

$$
\text { (J. K. Rowling, 1997) }
$$

"Ha-Li-Dui-Zhi-Zhu-Zao-Jiu-Xi-Guan-Le, Yin-Wei-Lou-Ti-Xia-Bian-De-Wan-Gui-LiDao-Chu-Dou-Shi-Zhi-Zhu."

(N. Su, 2009)

Cupboard means "a closet or cabinet, usually with shelves for storing food, crockery, and utensils". From the context it is found that by using 'Cupboard', the author refers to a place that can store bed and some furniture. It is not suitable for using "Wan-Gui", instead, the translation should be "Ha-Li-Dui-Zhi-Zhu-Zao-Jiu-Xi-Guan-Le, YinWei-Lou-Ti-Xia-Bian-De-Chu-Wu-Jian-Li-Dao-Chu-DouShi-Zhi-Zhu."

The third reason is the translation is too complicated that the target text is beyond the understanding of children. The following example shows the multi-negative or multiwords in translations.

\section{Example:}

"I'm not saying his heart isn't in the right place' said Professor McGonagall grudgingly 'but you can't pretend he's not careless. He tends to what was that?"

(J. K. Rowling, 1997)

"Wo-Bu-Shi-Shuo-Ta-Xin-Shu-Bu-Zheng,"

Mai-Ge-Jiao-Shou-Bu-Yi-Wei-Ran-De-Shuo,

"Ke-Shi-Nin-Bu-Neng-Bu-Kan-Dao-Ta-Hen-

$\mathrm{Cu}$-Xin. Ta-Zong-Shi...Na-Shi-Shen-Me-ShengYin?"

$$
\text { (N. Su, 2009) }
$$

In this example, both the source text and the target text are double negative. They seemingly accord with each other in form and style, but on the other hand the translation contains "Bu-Neng-Bu-Kan-Dao" in which "Bu-Neng-Bu" is used to modify "Kan-Dao". It is a little bit difficult for audience to understand. The Skopos theory lays emphasize on the feeling of the target audience, the equivalent is not so important it is obeyed with the Skopos rule. The translator should always give the audience high priority.

\section{Example:}

As he drove towards town he thought of nothing except a large order of drills he was hoping to get that day. But on the edge of town, drills were driven out of his mind by something.

$$
\text { (J. K. Rowling, 1997) }
$$

"Ta-Kai-Che-Jin-Cheng, Yi-Lu-Shang-
Xiang-De-Shi-Xi-Wang-Jin-Tian-Ta-Neng-Na-
Dao-Yi-Da-Pi-Zuan-Ji-De-Ding-Dan. Dan-Kuai-
in-Cheng-Shi, Ling-Yi-Jian-Shi-You-Ba-Zuan-
-De-Shi-Cong-Ta-Nao-Hai-Li-Gan-Zou-Le."

(N. Su, 2009)

As for this example, there are two "Shi" in the Chinese version "Ling-Yi-Jian-Shi-You-Ba-Zuan-Ji-De-Shi-CongTa-Nao-Hai-Li-Gan-Zou-Le.", which makes the version a little complicated. We could change it, as the context suggests, as "Dan-Kuai-Jin-Cheng-Shi, Ta-De-Nao-HaiYou-Xiang-Qi-Le-Qi-Ta-De-Shi”.

\section{CONCLUSIONS}

In conclusion, this paper shows the analysis of Harry Potter and the Philosopher's Stone from the aspect of 'Skopos theory'. For further study, there are three parts of work need to be done. Firstly, more detailed analysis based on the shortcomings of the translations needs to be done. For this part of work, it is advised to compare the translation of Chinese traditional version from Taiwan and Chinese Simplified version from mainland. After that, it is better to analyze the disadvantages of using 'Skopos theory' to translate the target text. The last work that needs to be done is to find the tips of translation by using 'Skopos theory'. For example, the translation of idiom or four-character words make the translation vivid.

\section{ACKNOWLEDGMENT}

This work is supported by the Fundamental Research Funds for the Central Universities of China for Northwest University for Nationalities (Grant No. 31920150007), the Zhejiang Open Foundation of the Most Important Subjects Program, the Project for Young Talents of State Ethnic Affairs Commission (SEAC) of China (Grant [2013] 231), and by National Natural Science Foundation (NNSF) of China (Grants 61263002 \& 61374054).

The authors would like to express thanks to Prof. M. J. Khan with the School of PN Engineering, National University of Sciences \& Technology, Islamabad, Pakistan and Prof. J. Cao with the Research Institute of Information Technology, Tsinghua University, and Beijing, China for the beneficial discussions about this interesting topic.

\section{REFERENCES}

[1] X. Wei, Children Literature Translation Under Perspective of Adaptive Theory, Shanxi Normal University, 2014.

[2] Y. Kang, Analysis of Mainland and Taiwan Versions of Harry Potter and Philosopher's Stone from Perspective of Subculture, Hebei Agricultural University, 2013. 
[3] Y. Wang and Z. Yue, "Spell translation in Harry Potter from the perspective of Skopostheorie: A comparative analysis on the versions from Mainland China and Taiwan", Studies in Literature and Language, vol. 7, pp. 119-124, 2013.

[4] K. Reiss, and H. J. Vermeer, Towards a General Theory of Translational Action: Skopos Theory Explained, Routledge, 2014.

[5] J. Munday, Introducing Translation Studies: Theories and Applications, Routledge, 2012.

[6] Y. Zhao and Y. Jiang, "Translation techniques of Chinese union version: From the perspective of Skopos theory", Canadian Social Science, vol. 10, pp. 9-13, 2014.

[7] L. Tack, "Review of translating as purposeful activity: functionalist approaches explained by Christiane Nord", Target, vol. 13, pp. 197-197, 2002.

[8] P. Flynn, "Skopos theory: An ethnographic enquiry", Perspectives, vol. 12, pp. 270-285, 2004.
[9] N. Jiang, On English-Chinese Children Literature Translation via Teleology: A Case Study of Chinese Version of Harry Potter From Perspective of Skopostheorie, Suzhou University, 2010.

[10] S. Wang, Translation Under a Context of Mass Culture, Hebei Normal University, 2008.

[11] C. A. Ellwood, An Introdution to Social Psychology, Hardpress Publishing, 2013.

[12] E. Sapir, Language-An Introduction to the Study of Speech, New York: Harcourt, Brace and Company, 1921.

[13] E. Sapir, "Culture, Genuine and Spurious", Am. J. Soc., vol. 29, pp. 401-429, 1924.

[14] L. Xue, "The application of traditional language learning strategy", J. Univ. Sci Tech. Beijing (Social Sciences Edition), vol. 6, pp. 114-116, 2008

[15] L. Xue, "The comparison between traditional Chinese literacy strategies and English vocabulary strategies", Sino-US English Teaching, vol. 1, pp. 42-45, 2009. 